\title{
Fluctuations of stable processes and exponential functionals of hypergeometric Lévy processes
}

\author{
A. Kuznetsov ${ }^{*}$, J. C. Pardo ${ }^{\dagger}$
}

Current version: May 29, 2018

\begin{abstract}
We study the distribution and various properties of exponential functionals of hypergeometric Lévy processes. We derive an explicit formula for the Mellin transform of the exponential functional and give both convergent and asymptotic series expansions of its probability density function. As applications we present a new proof of some of the results on the density of the supremum of a stable process, which were recently obtained in [25] and [23]. We also derive some new results related to (i) the entrance law of the stable process conditioned to stay positive, (ii) the entrance law of the excursion measure of the stable process reflected at its past infimum and (iii) the entrance law and the last passage time of the radial part of n-dimensional symmetric stable process.
\end{abstract}

Keywords: Hypergeometric Lévy processes, Lamperti-stable processes, exponential functional, double gamma function, Lamperti transformation, extrema of stable processes.

AMS 2000 subject classifications: 60G51, 60G52.

\section{Introduction}

Exponential functionals of Lévy processes play a very important role in various domains of probability theory, such as self-similar Markov processes, random processes in random environment, fragmentation processes, branching processes, mathematical finance, to name but a few. In general, the distribution of the exponential functional of a Lévy process $X=\left(X_{t}, t \geq 0\right)$ with lifetime $\zeta$, defined as

$$
I:=\int_{0}^{\zeta} e^{-X_{t}} \mathrm{~d} t
$$

can be rather complicated. Nonetheless, this distribution is known explicitly for the case when $X$ is either: a standard Poisson processes, Brownian motion with drift, a particular class of spectrally negative Lamperti stable (see for instance [18, 28, 34]), spectrally positive Lévy processes satisfying the Cramér condition (see for instance [36]). In the class of Lévy processes with double-sided jumps the

*Department of Mathematics and Statistics, York University, 4700 Keele Street, Toronto, Ontario, M3J 1P3, Canada. Email: kuznetsov@mathstat.yorku.ca. Research supported by the Natural Sciences and Engineering Research Council of Canada.

${ }^{\dagger}$ Centro de Investigación en Matemáticas A.C. Calle Jalisco s/n. 36240 Guanajuato, México. Email: jcpardo@cimat.mx 
distibution of the exponential functional is known in closed form only in the case of Lévy processes with hyperexponential jumps, see the recent paper by Cai and Kou [14]. An overview of this topic can be found in Bertoin and Yor [7].

For many applications, it is enough to have estimates of $\mathbb{P}(I<t)$ as $t \rightarrow 0^{+}$and $\mathbb{P}(I>t)$ as $t \rightarrow+\infty$. However it is quite difficult to obtain such estimates in the general case. The behaviour of the tail $\mathbb{P}(I>t)$ has been studied in a general setting (see for instance [18, 19, 22, 32, 37, 38]). On the other hand, the behaviour of $\mathbb{P}(I<t)$ has been studied only in two particular cases: when $X$ has exponential moments and its Laplace exponent is regularly varying at infinity with index $\theta \in(1,2)$ (see [33]) and when $X$ is a subordinator whose Laplace exponent is regularly varying at 0 (see [9]).

At the same time, the problem of finding the distribution of the supremum of a stable process has also stimulated a lot of research. The explcit expressions for the Wiener-Hopf factors for a dense set of parameters were first obtained by Doney [21]. In the spectrally positive case a convergent series representation for the density of supremum was first obtained by Bernyk, Dalang and Peskir [4], and a complete asymptotic expantion was derived by Patie [35]. The general case was treated recently in [25] and [23], where the authors have derived explicit formulas for the Wiener-Hopf factors, the Mellin transform of supremum and also convergent and asymptotic series representations for the density of the supremum.

In this paper we pursue two goals. First, we will study exponential functionals of hypergeometric processes, we will obtain the Mellin transform and both convergent and asymptotic series representations for the density of the exponential functional. This gives us the first explicit results on the exponential functionals of Lévy processes which have double-sided jumps of infinite activity or infinite variation. The technique that we develop to obtain these results may be of independent interest, as it provides a very simple and straightforward method to derive well-known results on the exponential functionals of Brownian motion with drift and the less-known (but very interesting) recent results by Cai and Kou [14] for processes with double-sided hyper-exponential jumps.

The second goal is to prove that hypergeometric processes include the Lamperti-stable processes, which will allow us to prove interesting results on fluctuations of stable processes with the help of the Lamperti transformation. In particular, we will obtain a new proof of some of the results in [25] and [23], which is more straightforward and less technical. We will also derive some new formulas related to the density of the entrance law of the excursion measure of the stable process reflected at its past infimum and will obtain some several results related to the $n$-dimensional symmetric stable process.

The paper is organized as follows: in Section 2 we introduce hypergeometric processes and establish the connections between this class and the Lamperti-stable processes. In Section 3 we study the Mellin transform of the exponential functional of the hypergeometric processes and in Section 4 we derive the convergent and asymptotic series representations for the density of the exponential functional. Finally, in Section 5 we present some applications of these results to fluctuations of stable processes.

\section{Hypergeometric and Lamperti-stable processes}

Hypergeometric processes were first introduced in [29] and, more generally, in [26]. These processes were originally constructed using Vigon's theory of philantropy (see [40]) and they provide examples of Lévy processes with an explicit Wiener-Hopf factorization. The class of processes which we will study in this paper should be considered as a generalization of the hypergeometric processes studied in [29] and of the Lamperti-stable processes, which were introduced by Caballero and Chaumont [10]. 
We start by defining a function $\psi(z)$ as

$$
\psi(z)=-\frac{\Gamma(1-\beta+\gamma-z)}{\Gamma(1-\beta-z)} \frac{\Gamma(\hat{\beta}+\hat{\gamma}+z)}{\Gamma(\hat{\beta}+z)}
$$

where $(\beta, \gamma, \hat{\beta}, \hat{\gamma})$ belong to the admissible set of parameters

$$
\mathcal{A}=\{\beta \leq 1, \gamma \in(0,1), \hat{\beta} \geq 0, \hat{\gamma} \in(0,1)\}
$$

Our first goal is to show that $\psi(z)$ is the Laplace exponent of a (possibly killed) Lévy process $X$, that is $\psi(z)=\ln \mathbb{E}\left[\exp \left(z X_{1}\right)\right]$. This process and its properties will be presented in the next Proposition. From now on we will use the following notation

$$
\eta=1-\beta+\gamma+\hat{\beta}+\hat{\gamma}
$$

\section{Proposition 1.}

(i) The function $\psi(z)$ defined by (1) is the Laplace exponent of a Lévy process $X$. The density of its Lévy measure is given by

$$
\pi(x)= \begin{cases}-\frac{\Gamma(\eta)}{\Gamma(\eta-\hat{\gamma}) \Gamma(-\gamma)} e^{-(1-\beta+\gamma) x_{2}} F_{1}\left(1+\gamma, \eta ; \eta-\hat{\gamma} ; e^{-x}\right), & \text { if } x>0, \\ -\frac{\Gamma(\eta)}{\Gamma(\eta-\gamma) \Gamma(-\hat{\gamma})} e^{(\hat{\beta}+\hat{\gamma}) x} F_{1}\left(1+\hat{\gamma}, \eta ; \eta-\gamma ; e^{x}\right), & \text { if } x<0,\end{cases}
$$

where ${ }_{2} F_{1}$ is the Gauss hypergeometric function.

(ii) When $\beta<1$ and $\hat{\beta}>0$ the process $X$ is killed at the rate

$$
q=-\psi(0)=\frac{\Gamma(1-\beta+\gamma)}{\Gamma(1-\beta)} \frac{\Gamma(\hat{\beta}+\hat{\gamma})}{\Gamma(\hat{\beta})}
$$

When $\beta=1$ and $\hat{\beta}>0\{\beta<1$ and $\hat{\beta}=0\} X$ drifts to $+\infty\{-\infty\}$ and

$$
\mathbb{E}\left[X_{1}\right]=\frac{\Gamma(\gamma) \Gamma(\hat{\beta}+\hat{\gamma})}{\Gamma(\hat{\beta})} \quad\left\{\mathbb{E}\left[X_{1}\right]=-\frac{\Gamma(\hat{\gamma}) \Gamma(1-\beta+\gamma)}{\Gamma(1-\beta)}\right\}
$$

When $\beta=1$ and $\hat{\beta}=0$ the process $X$ oscillates.

(iii) The process $X$ has no Gaussian component. When $\gamma+\hat{\gamma}<1\{1 \leq \gamma+\hat{\gamma}<2\}$ the process has paths of bounded variation and no linear drift \{paths of unbounded variation\}.

(iv) We have the Wiener-Hopf factorization $-\psi(z)=\kappa(q,-z) \hat{\kappa}(q, z)$ where

$$
\kappa(q, z)=\frac{\Gamma(1-\beta+\gamma+z)}{\Gamma(1-\beta+z)}, \quad \hat{\kappa}(q, z)=\frac{\Gamma(\hat{\beta}+\hat{\gamma}+z)}{\Gamma(\hat{\beta}+z)}
$$

(v) The process $\hat{X}=-X$ is a hypergeometric process with parameters $(1-\hat{\beta}, \hat{\gamma}, 1-\beta, \gamma)$. 
Proof. First let us prove (i). Let $X^{(1)}$ be a general hypergeometric Lévy process (see section 3.2 in [26]) with parameters

$$
\begin{aligned}
& \sigma=d=k_{1}=\delta_{1}=\delta_{2}=0, \beta=1, c_{1}=-\frac{1}{\Gamma(-\gamma)}, c_{2}=-\frac{1}{\Gamma\left(-\hat{\gamma}_{2}\right)} \\
& \alpha_{1}=\beta, \alpha_{2}=1-\hat{\beta}, \gamma_{1}=\gamma, \gamma_{2}=\hat{\gamma}, k_{2}=\frac{\Gamma(\hat{\beta}+\hat{\gamma})}{\Gamma(\hat{\beta})} .
\end{aligned}
$$

This process is constructed using Vigon's theory of philantropy (see [40]) from two subordinators $H^{(1)}$ and $\hat{H}^{(1)}$ which have Laplace exponents

$$
\kappa^{(1)}(q, z)=\kappa(q, z)-\kappa(q, 0), \quad \hat{\kappa}^{(1)}(q, z)=\hat{\kappa}(q, z)
$$

where $\kappa(q, z)$ and $\hat{\kappa}(q, z)$ are given by (5). We see that the Lapalace exponent $\psi^{(1)}(z)$ of process $X^{(1)}$ satisfies

$$
\psi^{(1)}(z)=\psi(z)+k \hat{\kappa}(q, z)
$$

where we have denoted $k=\kappa(q, 0)$. Therefore the process $X_{t}^{(1)}$ has the same distribution as $X_{t}-\hat{H}_{k t}$, in particular the distribution of positive jumps of $X^{(1)}$ coincides with the distribution of positive jumps of $X$. From [26] we find that the Lévy measure of $X^{(1)}$ restricted to $x>0$ coincides with (4). The expression of the Lévy measure for $x<0$ follows easily by symmetry considerations. This proves that $\psi(z)$ defined by (1) is the Laplace transform of a (possibly killed) Lévy process with the density of the Lévy measure given by (4).

The rest of the proof is rather straightforward. Property (v) follows easily from the definition of the Laplace exponent (1). The Wiener-Hopf factorization (iv) follows easily by construction: we know that both $\kappa(q, z)$ and $\hat{\kappa}(q, z)$ defined by (5) are Laplace transforms of (possibly killed) subordinators, and the result follows from identity $-\psi(z)=\kappa(q,-z) \hat{\kappa}(q, z)$ and the uniqueness of the Wiener-Hopf factorization (see $[27])$.

Let us prove (ii). The fact that $X$ drifts to $+\infty$ when $\beta=1$ and $\hat{\beta}>0$ follows from the Wiener-Hopf factorization (iv): in this case $\kappa(q, 0)=0$, therefore the ascending ladder height process drifts to $+\infty$, while the descending ladder height process is killed at a rate $\hat{\kappa}(q, 0)>0$. The expression for $\mathbb{E}\left[X_{1}\right]$ follows from (1) using the fact that $\mathbb{E}\left[X_{1}\right]=\psi^{\prime}(0)$. Other results in (ii) can be verified in a similar way.

Let us prove (iii). Formula (1) and the asymptotic expansion for the Gamma function (see formula 8.328.1 in [24]) imply that

$$
\psi(\mathrm{i} z)=O\left(|z|^{\gamma+\hat{\gamma}}\right)=o\left(|z|^{2}\right), \quad z \rightarrow \infty, z \in \mathbb{R} .
$$

Applying Proposition 2 from [5] we conclude that $X$ has no Gaussian component, and that when $\gamma+\hat{\gamma}<1$ $\{1<\gamma+\hat{\gamma}<2\}$ the process has paths of bounded variation and no linear drift \{paths of unbounded variation $\}$. In the remaining case $\gamma+\hat{\gamma}=1$ the density of the Lévy measure has a singularity of the form $C x^{-2}+o\left(x^{-2}\right)$ as $x \rightarrow 0^{+}$(see formula 15.3.12 in [1]), which implies that the process has paths of unbounded variation.

Remark 1: It is possible to extend the definition of the hypergeometric processes to the case when $\gamma \in\{0,1\}$ or $\hat{\gamma} \in\{0,1\}$. In the case when $\gamma=1$ and $\hat{\gamma} \in(0,1)$ the process $X$ is spectrally negative. This is true due to the formula (4), which shows that $\pi(x)=0$ for $x>0$; one can also establish this 
fact using (5), which imlies that $\kappa(q, z)=1-\beta+z$. Similarly, when $\hat{\gamma}=1$ and $\gamma \in(0,1)$ the process $X$ is spectrally positive. When $\gamma=\hat{\gamma}=1$ the process $X$ is Brownian motion with drift, killed at an exponential time. When $\gamma=0\{\hat{\gamma}=0\}$ the process $-X\{X\}$ is a subordinator.

The three Lamperti-stable processes $\xi^{*}, \xi^{\uparrow}$ and $\xi^{\downarrow}$ were introduced by Caballero and Chaumont [10] by applying the Lamperti transformation (see [30]) to the positive self-similar Markov processes related to stable process. In particular, the process $\xi^{*}$ is obtained from a stable process started at $x>0$ and killed on the first exit from the positive half-line, while the process $\xi^{\uparrow}\left\{\xi^{\downarrow}\right\}$ is obtained from stable process conditioned to stay positive $\{$ conditioned to hit zero continuously $\}$. We refer to $[10,18,13]$ for all the details on these processes.

For our next result we will need the form of the Laplace exponent of the Lamperti-stable process $\xi^{*}$. We assume that the characteristic exponent $\Psi_{Y}(z)=-\ln \mathbb{E}\left[\exp \left(\mathrm{i} z Y_{1}\right)\right]$ of a stable process $Y$ is defined as

$$
\Psi_{Y}(z)=e^{\frac{\pi \mathrm{i} \gamma}{2}} z^{\alpha} \mathbf{1}_{\{z>0\}}+e^{-\frac{\pi \mathrm{i} \gamma}{2}}|z|^{\alpha} \mathbf{1}_{\{z<0\}},
$$

where $|\gamma|<\alpha$ if $\alpha \in(0,1)$ and $|\gamma|<1$ if $\alpha \in(1,2)$. With this parametrization the density of the Lévy measure of $Y$ is given by

$$
\pi_{Y}(x)=c_{+} x^{-1-\alpha} \mathbf{1}_{\{x>0\}}+c_{-}|x|^{-1-\alpha} \mathbf{1}_{\{x<0\}},
$$

where

$$
c_{+}=\Gamma(1+\alpha) \frac{\sin (\pi \alpha \rho)}{\pi}, \quad c_{-}=\Gamma(1+\alpha) \frac{\sin (\pi \alpha(1-\rho))}{\pi}
$$

and $\rho=\mathbb{P}\left(Y_{1}>0\right)=(1-\gamma / \alpha) / 2$. Note that this parameter was corresponds to $1-\rho$ in $[10]$. According to Caballero and Chaumont [10], the Laplace exponent $\psi^{*}(z)=\ln \mathbb{E}\left[\exp \left(z \xi_{1}^{*}\right)\right]$ of the Lamperti-stable process $\xi^{*}$ is given by

$$
\psi^{*}(z)=\frac{c_{+}-c_{-}}{1-\alpha} z+\int_{\mathbb{R} \backslash\{0\}}\left(e^{z x}-1-z\left(e^{x}-1\right) 1_{\left\{\left|e^{x}-1\right|<1\right\}}\right) e^{x} \pi_{Y}\left(e^{x}-1\right) \mathrm{d} x-c_{-} \alpha^{-1} .
$$

It is important to note that when $\alpha<1$, the Laplace exponent (9) can be rewritten as

$$
\psi^{*}(z)=\int_{\mathbb{R} \backslash\{0\}}\left(e^{z x}-1\right) e^{x} \pi_{Y}\left(e^{x}-1\right) \mathrm{d} x-c_{-} \alpha^{-1},
$$

so that in this case $\xi^{*}$ is a process of bounded variation with no linear drift.

Theorem 1. Lamperti-stable processes $\xi^{*}, \xi^{\uparrow}, \xi^{\downarrow}$ can be identified as hypergeometric processes with the following sets of parameters

\begin{tabular}{|c||c|c|c|c|}
\hline & $\beta$ & $\gamma$ & $\hat{\beta}$ & $\hat{\gamma}$ \\
\hline \hline$\xi^{*}$ & $1-\alpha(1-\rho)$ & $\alpha \rho$ & $1-\alpha(1-\rho)$ & $\alpha(1-\rho)$ \\
\hline$\xi^{\uparrow}$ & 1 & $\alpha \rho$ & 1 & $\alpha(1-\rho)$ \\
\hline$\xi^{\downarrow}$ & 0 & $\alpha \rho$ & 0 & $\alpha(1-\rho)$ \\
\hline
\end{tabular}


Proof. For the proof of the result for $\xi^{\uparrow}$ see Proposition 2 in [29]. The result for $\xi^{\downarrow}$ follows from Proposition 1 in [18]. Thus we only need to prove the result for $\xi^{*}$.

Let us set $(\beta, \gamma, \hat{\beta}, \hat{\gamma})=(1-\alpha(1-\rho), \alpha \rho, 1-\alpha(1-\rho), \alpha(1-\rho))$ and compute the Lévy measure of the hypergeometric process $X$. We find that $\eta=1+\alpha$, and formula (4) implies that for $x>0$ we have

$$
\begin{aligned}
\pi(x) & =-\frac{\Gamma(1+\alpha)}{\Gamma(1+\alpha \rho) \Gamma(-\alpha \rho)} e_{2}^{-\alpha x} F_{1}\left(1+\alpha \rho, 1+\alpha ; 1+\alpha \rho ; e^{-x}\right) \\
& =\Gamma(1+\alpha) \frac{\sin (\pi \alpha \rho)}{\pi} e^{-\alpha x}\left(1-e^{-x}\right)^{-1-\alpha}=e^{x} \pi_{Y}\left(e^{x}-1\right)
\end{aligned}
$$

where we have used the reflection formula for the gamma function and the fact that ${ }_{2} F_{1}(a, b ; a, z)=$ $(1-z)^{-b}$. Similarly, we find that $\pi(x)=e^{x} \pi_{Y}\left(e^{x}-1\right)$ for $x<0$. We see that the Lévy measure of the hypergeometric process is the same as the Lévy measure of the Lamperti-stable process. We know that in the case $\alpha<1$ both of these processes have paths of finite variation and no linear drift, this proves that $X \stackrel{d}{=} \xi^{*}$ in the case $\alpha<1$.

In the case $\alpha>1$ processes $X$ and $\xi^{*}$ have infinite variation, no Gaussian component and identical Lévy measures. Thus their Laplace exponents may differ only by a linear function. Thus in order to establish that the Laplace exponents are equal it is enough to show that $\mathbb{E}\left[X_{1} \mid \zeta>1\right]=\mathbb{E}\left[\xi_{1}^{*} \mid \zeta>1\right]$. Using (1) and the reflection formula for the gamma function we find that the Laplace exponent of the hypergeometric process $X$ is given by

$$
\psi_{X}(z)=\frac{1}{\pi} \Gamma(\alpha-z) \Gamma(1+z) \sin (\pi(z-\alpha(1-\rho)))
$$

Therefore we have

$$
\mathbb{E}\left[X_{1} \mid \zeta>1\right]=\psi_{X}^{\prime}(0)=\Gamma(\alpha) \frac{\sin (\pi \alpha(1-\rho))}{\pi}(\Psi(\alpha)-\Psi(1))+\Gamma(\alpha) \cos (\pi \alpha(1-\rho)) .
$$

where $\Psi(z)=\Gamma^{\prime}(z) / \Gamma(z)$ is the digamma function. On the other hand, equation (11) in [10] tells us that

$$
\begin{gathered}
\mathbb{E}\left[\xi_{1}^{*} \mid \zeta>1\right]=\frac{c_{+}-c_{-}}{1-\alpha}+c_{+}\left[\int_{0}^{\ln (2)} \frac{\left(1+y-e^{y}\right) e^{y}}{\left(e^{y}-1\right)^{1+\alpha}} \mathrm{d} y+\int_{\ln (2)}^{\infty} \frac{y e^{y}}{\left(e^{y}-1\right)^{1+\alpha}} \mathrm{d} y\right] \\
+c_{-} \int_{-\infty}^{0} \frac{\left(1+y-e^{y}\right) e^{y}}{\left(1-e^{y}\right)^{1+\alpha}} \mathrm{d} y
\end{gathered}
$$

where $c_{+}$and $c_{-}$are defined in (8). Let us show that the expressions in the right-hand side of (10) and (11) are equal.

First we will deal with the integrals multiplying $c^{+}$in (11). We rearrange the terms as follows

$$
\int_{0}^{\ln (2)} \frac{\left(1+y-e^{y}\right) e^{y}}{\left(e^{y}-1\right)^{1+\alpha}} \mathrm{d} y+\int_{\ln (2)}^{\infty} \frac{y e^{y}}{\left(e^{y}-1\right)^{1+\alpha}} \mathrm{d} y=\int_{0}^{\infty} \frac{\left(1+y-e^{y}\right) e^{y}}{\left(e^{y}-1\right)^{1+\alpha}} \mathrm{d} y+\int_{\ln (2)}^{\infty} \frac{\left(e^{y}-1\right) e^{y}}{\left(e^{y}-1\right)^{1+\alpha}} \mathrm{d} y
$$

Performing the change of variables $u=\exp (y)$ it is easy to see that the second integral in the right-hand side of $(12)$ is equal to $1 /(\alpha-1)$. In order to compute the first integral we use integration by parts

$$
\int_{0}^{\infty} \frac{\left(1+y-e^{y}\right) e^{y}}{\left(e^{y}-1\right)^{1+\alpha}} \mathrm{d} y=-\frac{1}{\alpha} \int_{0}^{\infty}\left(1+y-e^{y}\right) \mathrm{d}\left(e^{y}-1\right)^{-\alpha}=\frac{1}{\alpha} \int_{0}^{\infty} \frac{1-e^{y}}{\left(e^{y}-1\right)^{\alpha}} \mathrm{d} y=\frac{\pi}{\alpha \sin (\pi \alpha)}
$$


where in the last step we have used the following integral formula

$$
\int_{0}^{\infty} \frac{e^{a u}}{\left(e^{b u}-1\right)^{c}} \mathrm{~d} u=\frac{1}{b} \frac{\Gamma\left(c-\frac{a}{b}\right) \Gamma(1-c)}{\Gamma\left(1-\frac{a}{b}\right)}, \quad \frac{a}{b}<c<1,
$$

and the reflection formula for the gamma function. The last integral in (11) can also be computed using integration by parts

$$
\begin{aligned}
\int_{-\infty}^{0} \frac{\left(1+y-e^{y}\right) e^{y}}{\left(1-e^{y}\right)^{1+\alpha}} \mathrm{d} y=\int_{0}^{\infty} \frac{\left(1-y-e^{-y}\right) e^{\alpha y}}{\left(e^{y}-1\right)^{1+\alpha}} \mathrm{d} y \\
=-\frac{1}{\alpha} \int_{0}^{\infty}\left(1-y-e^{-y}\right) e^{(\alpha-1) y} \mathrm{~d}\left(e^{y}-1\right)^{-\alpha} \\
=\frac{1}{\alpha} \int_{0}^{\infty} \frac{\left(-1+e^{-y}\right) e^{(\alpha-1) y}+\left(1-y-e^{-y}\right)(\alpha-1) e^{(\alpha-1) y}}{\left(e^{y}-1\right)^{\alpha}} \mathrm{d} y \\
=\frac{\alpha-2}{\alpha} \int_{0}^{\infty} \frac{e^{(\alpha-2) y}}{\left(e^{y}-1\right)^{\alpha-1}} \mathrm{~d} y-\frac{\alpha-1}{\alpha} \int_{0}^{\infty} \frac{y e^{(\alpha-1) y}}{\left(e^{y}-1\right)^{\alpha}} \mathrm{d} y \\
=\frac{\alpha-2}{\alpha} \mathrm{B}(2-\alpha, 1)-\frac{\alpha-1}{\alpha} \frac{\mathrm{d}}{\mathrm{d} z}\left[\int_{0}^{\infty} \frac{e^{z y}}{\left(e^{y}-1\right)^{\alpha}} \mathrm{d} y\right]_{z=\alpha-1} \\
=-\frac{1}{\alpha}-\frac{1}{\alpha}(\Psi(1)-\Psi(2-\alpha))
\end{aligned}
$$

where in the last step we have again used (14). Combining (11), (12), (13) and (15) we see that

$$
\mathbb{E}\left[\xi_{1}^{*} \mid \zeta>1\right]=\frac{c_{+}-c_{-}}{1-\alpha}+c_{+}\left[\frac{1}{\alpha-1}+\frac{\pi}{\alpha \sin (\pi \alpha)}\right]+c_{-}\left[-\frac{1}{\alpha}-\frac{1}{\alpha}(\Psi(1)-\Psi(2-\alpha))\right] .
$$

Using the fact that $\Psi(2-\alpha)=\Psi(\alpha)+\pi \cot (\pi \alpha)+1 /(1-\alpha)$, the identity $\Gamma(z+1)=z \Gamma(z)$ and addition formula for the sine function it is not hard to reduce the above expression to (10), we leave all the details to the reader.

\section{Mellin transform of the exponential functional}

Let $X$ be the hypergeometric Lévy process with parameters $(\beta, \gamma, \hat{\beta}, \hat{\gamma}) \in \mathcal{A}$ and $\hat{\beta}>0$. We assume that $\alpha>0$ and define the exponential functional as

$$
I(\alpha, X)=\int_{0}^{\zeta} e^{-\alpha X_{t}} \mathrm{~d} t
$$


where $\zeta$ is the lifetime of the process $X$. Note that the above integral converges with probability one: according to Proposition 1, either $\zeta$ is finite (if $\beta<1$ ) or $\zeta=+\infty$ and the process $X$ drifts to $+\infty$ (if $\beta=1)$.

Our main tool in studying the exponential functional is the Mellin transform, which is defined as

$$
\mathcal{M}(s)=\mathcal{M}(s ; \alpha, \beta, \gamma, \hat{\beta}, \hat{\gamma})=\mathbb{E}\left[I(\alpha, X)^{s-1}\right] .
$$

From the definition of the Laplace exponent (1) we find that $X$ satisfies Cramér's condition $\mathbb{E}\left[\exp \left(-\hat{\beta} X_{1}\right)\right]=$ 1 , therefore applying Lemma 2 from [39] we conclude that $\mathcal{M}(s)$ exists for $s \in(0,1+\hat{\beta} \delta)$.

In order to describe our main result in this section, we need to define the Barnes double gamma function, $G(z ; \tau)$. This function was introduced by Alexeiewsky in 1889 and was extensively studied by Barnes in [2] and [3]. The double gamma function is defined as an infinite product in Weierstrass's form

$$
G(z ; \tau)=\frac{z}{\tau} e^{a \frac{z}{\tau}+b \frac{z^{2}}{2 \tau}} \prod_{m \geq 0} \prod_{n \geq 0}^{\prime}\left(1+\frac{z}{m \tau+n}\right) e^{-\frac{z}{m \tau+n}+\frac{z^{2}}{2(m \tau+n)^{2}}}, \quad|\arg (\tau)|<\pi, \quad z \in \mathbb{C} .
$$

Here the prime in the second product means that the term corresponding to $m=n=0$ is omitted. Note that by definition $G(z ; \tau)$ is an entire function in $z$ and has simple zeros on the lattice $m \tau+n, m \leq 0$, $n \leq 0$. The function $G(z ; \tau)$ can also be expressed as an infinite product of gamma functions, see [2]. An integral representation for $\ln (G(z ; \tau))$ was obtained in [31] and several important asymptotic expansions were established in [8].

It turns out that it is possible to define constants $a=a(\tau)$ and $b=b(\tau)$ in a particular way, so that we have $G(1 ; \tau)=1$ and that $G(z ; \tau)$ satisfies the following three functional identities (see [2] and [25])

$$
\begin{aligned}
G(z+1 ; \tau) & =\Gamma\left(\frac{z}{\tau}\right) G(z ; \tau) \\
G(z+\tau ; \tau) & =(2 \pi)^{\frac{\tau-1}{2}} \tau^{-z+\frac{1}{2}} \Gamma(z) G(z ; \tau) \\
G(z ; \tau) & =(2 \pi)^{\frac{z}{2}\left(1-\frac{1}{\tau}\right)} \tau^{-\frac{z^{2}}{2 \tau}+\frac{z}{2}\left(1+\frac{1}{\tau}\right)-1} G\left(\frac{z}{\tau} ; \frac{1}{\tau}\right)
\end{aligned}
$$

Next we introduce a function which will play a central role in our study of exponential functionals. Everywhere in this paper we will use notation

$$
\delta=\frac{1}{\alpha}
$$

Definition 1. For $s \in \mathbb{C}$ we define

$$
M(s)=M(s ; \alpha, \beta, \gamma, \hat{\beta}, \hat{\gamma})=C \frac{G((1-\beta) \delta+s ; \delta)}{G((1-\beta+\gamma) \delta+s ; \delta)} \frac{G((\hat{\beta}+\hat{\gamma}) \delta+1-s ; \delta)}{G(\hat{\beta} \delta+1-s ; \delta)}, \quad s \in \mathbb{C}
$$

where the constant $C$ is such that $M(1)=1$.

Our main result in this section is the following Theorem, which provides an explicit expression for the Mellin transform of the exponential functional.

Theorem 2. Assume that $\alpha>0,(\beta, \gamma, \hat{\beta}, \hat{\gamma}) \in \mathcal{A}$ and $\hat{\beta}>0$. Then $\mathcal{M}(s) \equiv \Gamma(s) M(s)$ for all $s \in \mathbb{C}$.

Before we are able to prove Theorem 2, we need to establish several auxiliary results. 


\section{Lemma 1.}

(i) Assume that $\tau>0$. When $s \rightarrow \infty$ in the domain $|\arg (s)|<\pi-\epsilon<\pi$ we have

$$
\ln \left[\frac{G(a+s ; \tau)}{G(s ; \tau)}\right]=\frac{a}{\tau} s \ln (s)-\frac{a}{\tau}(1+\ln (\tau)) s+\frac{a}{2 \tau}(a-1-\tau) \ln (s)+O(1) .
$$

(iv) When $s \rightarrow \infty$ in the domain $0<\epsilon<\arg (s)<\pi-\epsilon$ we have

$$
\ln (M(s))=-(\gamma+\hat{\gamma}) s \ln (s)+s((1+\ln (\delta))(\gamma+\hat{\gamma})+\pi \mathrm{i} \hat{\gamma})+O(\ln (s))
$$

(iii) When $s \rightarrow \infty$ in a vertical strip $a<\operatorname{Re}(s)<b$ we have

$$
|M(s)|=\exp \left(\frac{\pi}{2}(\gamma-\hat{\gamma})|\operatorname{Im}(s)|+O(\ln |\operatorname{Im}(s)|)\right) .
$$

Proof. Part (i) follows from the asymptotic expansion for $G(z ; \tau)$, given in formula (4.5) in [8], while parts (ii) and (iii) are simple corollaries of (i) and (22).

The following notation will be used extensively in this paper: if $X$ is a hypergeometric process with parameters $(\beta, \gamma, \hat{\beta}, \hat{\gamma})$, then $\tilde{X}$ denotes the hypergeometric process with parameters $(\delta \beta, \delta \gamma, \delta \hat{\beta}, \delta \hat{\gamma})$, provided that this parameter set is admissible. In particular, the Laplace exponent of $\tilde{X}$ is given by

$$
\tilde{\psi}(z)=-\frac{\Gamma(1-\delta(\beta-\gamma)-z) \Gamma(\delta(\hat{\beta}+\hat{\gamma})+z)}{\Gamma(1-\delta \beta-z) \Gamma(\delta \hat{\beta}+z)} .
$$

\section{Lemma 2.}

(i) $M(s)$ is a real meromorphic function which has zeros

$$
\{-(1-\beta) \delta-m \delta-n, \quad 1+(\hat{\beta}+\hat{\gamma}) \delta+m \delta+n\}_{m, n \geq 0},
$$

and poles

$$
\left\{z_{m, n}^{-}=-(1-\beta+\gamma) \delta-m \delta-n, \quad z_{m, n}^{+}=1+\hat{\beta} \delta+m \delta+n\right\}_{m, n \geq 0}
$$

All zeros/poles are simple if $\alpha \notin \mathbb{Q}$.

(ii) $M(s)$ satisfies the following functional identities

$$
\begin{aligned}
M(s+1) & =-\frac{1}{\psi(-\alpha s)} M(s), \\
M(s+\delta) & =-\frac{\alpha^{-\delta(\hat{\gamma}+\gamma)}}{\tilde{\psi}(1-\delta-s)} M(s), \\
M(s ; \alpha, \beta, \gamma, \hat{\beta}, \hat{\gamma}) & =\alpha^{(1-s)(\gamma+\hat{\gamma})} M(1-\alpha+\alpha s ; \delta, \delta \beta, \delta \gamma, \delta \hat{\beta}, \delta \hat{\gamma}) .
\end{aligned}
$$


Proof. The proof of (i) follows from the definition of the double gamma function (18), while functional identity (29) is a simple corollary of the functional identity for the double gamma function (19).

Let us prove (31). We use (21) and find that for all $s$ and $x$

$$
\frac{G(s+x ; \delta)}{G(s ; \delta)}=\alpha^{\alpha s x} C \frac{G(\alpha(s+x) ; \alpha)}{G(\alpha s ; \alpha)}
$$

where $C=C(x)$ depends only on $x$. The above identity implies that

$$
\begin{aligned}
& \frac{G((1-\beta) \delta+s ; \delta)}{G((1-\beta+\gamma) \delta+s ; \delta)} \frac{G((\hat{\beta}+\hat{\gamma}) \delta+1-s ; \delta)}{G(\hat{\beta} \delta+1-s ; \delta)} \\
& =\tilde{C} \alpha^{-s(\gamma+\hat{\gamma})} \frac{G(1-\beta+\alpha s ; \alpha)}{G(1-\beta+\gamma+\alpha s ; \alpha)} \frac{G(\hat{\beta}+\hat{\gamma}+\alpha-\alpha s ; \alpha)}{G(\hat{\beta}+\alpha-\alpha s ; \alpha)}
\end{aligned}
$$

where $\tilde{C}$ does not depend on $s$. It is easy to see that (31) follows from the above identity and (22).

The functional identity (30) follows from (31) and (29), we leave all the details to the reader.

The following proposition will be central in the proof of Theorem 2. It allows us to identify explicitly the Mellin transform of the exponential functional. This result is also applicable to some other Lévy process, including Brownian motion with drift and more generally, processes with hyperexponential or phase-type jumps, therefore it is of independent interest.

First let us present the main ingredients. Let $Y$ be a (possibly killed) Lévy process started from zero, and let $\psi_{Y}(z)=\ln \mathbb{E}\left[\exp \left(z Y_{1}\right)\right]$ denote its Laplace exponent. In the case when $\psi_{Y}(0)=0$ (the process is not killed) we will also assume that $\mathbb{E}\left[Y_{1}\right]>0$, so that $Y$ drifts to $+\infty$. As usual we define the exponential functional $I=\int_{0}^{\zeta} \exp \left(-Y_{t}\right) \mathrm{d} t$ (where $\zeta$ is the lifetime of $Y$ ) and the Mellin transform $\mathcal{M}_{Y}(s)=\mathbb{E}\left[I^{s-1}\right]$.

Proposition 2. (Verification result) Assume that Cramér's condition is satisfied: there exists $z_{0}<0$ such that $\psi_{Y}(z)$ is finite for all $z \in\left(z_{0}, 0\right)$ and $\psi_{Y}(-\theta)=0$ for some $\theta \in\left(0,-z_{0}\right)$. If $f(s)$ satisfies the following three properties

(i) $f(s)$ is analytic and zero-free in the strip $\operatorname{Re}(s) \in(0,1+\theta)$,

(ii) $f(1)=1$ and $f(s+1)=-s f(s) / \psi_{Y}(-s)$ for all $s \in(0, \theta)$,

(iii) $|f(s)|^{-1}=o(\exp (2 \pi|\operatorname{Im}(s)|))$ as $\operatorname{Im}(s) \rightarrow \infty, \operatorname{Re}(s) \in(0,1+\theta)$,

then $\mathcal{M}_{Y}(s) \equiv f(s)$ for $\operatorname{Re}(s) \in(0,1+\theta)$.

Proof. The Cramér's condition and Lemma 2 in [39] imply that $\mathcal{M}_{Y}(s)$ can be extended to an analytic function in the strip $\operatorname{Re}(s) \in(0,1+\theta)$. In the case when $\psi_{Y}(0)=0\left\{\psi_{Y}(0)<0\right\}$ we use Lemma 2.1 in [32] $\{$ Proposition 3.1 from $[15]\}$ to conclude that $\mathcal{M}_{Y}(s)$ satisfies the functional identity

$$
\mathcal{M}_{Y}(s+1)=-\frac{s}{\psi_{Y}(-s)} \mathcal{M}_{Y}(s)
$$

for all $s \in(0, \theta)$. Since $f(s)$ satisfies the same functional identity we conclude that the function $F(s)=$ $\mathcal{M}_{Y}(s) / f(s)$ satisfies $F(s+1)=F(s)$ for all $s \in(0, \theta)$. Using the assumption that $f(s)$ is analytic and 
zero-free we conclude that $F(s)$ is an analytic function in the strip $\operatorname{Re}(s) \in(0,1+\theta)$. Since $F(s)$ is also periodic with period equal to one, it can be extended to an analytic and periodic function in the entire complex plane.

Our goal now is to prove that function $F(s)$ is constant. Since $F(s)$ is analytic and periodic in the entire complex plane, it can be represented as a Fourier series

$$
F(s)=c_{0}+F_{1}(\exp (2 \pi \mathrm{i} s))+F_{2}(\exp (-2 \pi i s)),
$$

where $F_{i}(z)$ are entire functions

$$
F_{1}(z)=\sum_{n \geq 1} c_{n} z^{n}, \quad F_{2}(z)=\sum_{n \geq 1} c_{-n} z^{n}
$$

Due to the inequality $\left|\mathcal{M}_{Y}(s)\right|<\mathcal{M}_{Y}(\operatorname{Re}(s))$ and assumption (iii) we conclude that $F(s)=o(\exp (2 \pi|\operatorname{Im}(s)|))$ as $\operatorname{Im}(s) \rightarrow \infty$. As $\operatorname{Im}(s) \rightarrow-\infty$ this estimate and equation $(33)$ imply that $F_{1}(z)=o(z)$ as $z \rightarrow \infty$, and using Cauchy's estimates (Proposition 2.14 in [20]) we conclude that $F_{1}(z) \equiv 0$. Similarly we find that $F_{2}(z) \equiv 0$, thus $F(s)$ is a constant, and the value of this constant is one, since $\mathcal{M}_{Y}(1)=f(1)=1$.

We would like to stress that Proposition 2 is an important result of indepent interest. We know that if Cramér's condition is satisfied then the Mellin transform $\mathcal{M}_{Y}(s)$ satisfies the functional identity (32), however it is clear that there are infinitely many functions which satisfy the same functional identity. Proposition 2 tells us that if we have found such a function $f(s)$, which satisfies (32), and if we can verify the two conditions about the zeros of this function and its asymptotic behaviour, then we can in fact uniquely identify $\mathcal{M}_{Y}(s) \equiv f(s)$. In particular, this proposition can be used to provide a very simple and short proof of the well-known result on exponential functional of Brownian motion with drift and of the recent results on exponential functionals of processes with double-sided hyper-exponential jumps (see [14]).

Proof of Theorem 2: First of all, we check that the Cramér's condition is satisfied with $\theta=\hat{\beta} \delta$. Let $f(s)=\Gamma(s) M(s)$, where $M(s)$ is defined by (22). From Lemma 2(i) we know that $f(s)$ is analytic and zero-free in the strip $\operatorname{Re}(s) \in(0,1+\hat{\beta} \delta)$. By construction we have $f(1)=1$, and from the formula (29) we find that $f(s)$ satisfies $f(s+1)=-s f(s) / \psi(-\alpha s)$ for $s \in(0, \hat{\beta} \delta)$. Next, Lemma 1 (iii) and Stirling's asymptotic formula for the gamma function (see formula 8.327.3 in [24])

$$
\ln (\Gamma(s))=s \ln (s)-s+O(\ln (s))
$$

imply that as $s \rightarrow \infty$ in the vertical strip $\operatorname{Re}(s) \in(0,1+\hat{\beta} \delta)$ we have

$$
|f(s)|^{-1}=\exp \left(\frac{\pi}{2}(1-\gamma+\hat{\gamma})|\operatorname{Im}(s)|+o(\operatorname{Im}(s))\right)=o(\exp (\pi|\operatorname{Im}(s)|)),
$$

where in the last step we have also used the fact that both $\gamma$ and $\hat{\gamma}$ belong to the interval $(0,1)$.

We see that function $f(s)$ satisfies all conditions of Proposition 2, thus we can conclude that $\mathcal{M}(s) \equiv$ $f(s)$.

Corollary 1. Assume that $\alpha>0, \hat{\beta}>0$ and that both sets of parameters $(\beta, \gamma, \hat{\beta}, \hat{\gamma})$ and $(\delta \beta, \delta \gamma, \delta \hat{\beta}, \delta \hat{\gamma})$ belong to the admissible set $\mathcal{A}$. Then we have the following identity in distribution

$$
\epsilon_{1}^{\alpha} \times I(\alpha ; X) \stackrel{d}{=} \alpha^{\gamma+\hat{\gamma}} \times \epsilon_{1} \times I(\delta ; \tilde{X})^{\alpha}
$$

where $\epsilon_{1} \sim \operatorname{Exp}(1)$ and all random variables are assumed to be independent. 
Proof. Rewrite (31) as

$$
\Gamma(1-\alpha+\alpha s) \mathcal{M}(s ; \alpha, \beta, \gamma, \hat{\beta}, \hat{\gamma})=\alpha^{(s-1)(\gamma+\hat{\gamma})} \Gamma(s) \mathcal{M}(1-\alpha+\alpha s ; \delta, \delta \beta, \delta \gamma, \delta \hat{\beta}, \delta \hat{\gamma})
$$

and use the following facts: (i) $\Gamma(s)=\mathbb{E}\left[\epsilon_{1}^{s-1}\right]$; (ii) if $f(s)$ is the Mellin transform of a random variable $\xi$ then $f(1-\alpha+\alpha s)$ is the Mellin transform of the random variable $\xi^{\alpha}$; (iii) Mellin transform of the product of independent random variables is the product of their Mellin transforms.

\section{Density of the exponential functional}

In this section we will study the density of the exponential functional, defined as

$$
p(x)=\frac{\mathrm{d}}{\mathrm{d} x} \mathbb{P}(I(\alpha, X) \leq x), \quad x \geq 0 .
$$

As in the previous section, $X$ is a hypergeometric process with parameters $(\beta, \gamma, \hat{\beta}, \hat{\gamma}) \in \mathcal{A}$ and $\hat{\beta}>0$. The main results of this section are the convergent series representations and complete asymptotic expansions of this function as $x \rightarrow 0^{+}$or $x \rightarrow+\infty$.

Let us define the following three sets of parameters which will be used extensively later. In the following definition (and everywhere else in this paper) $\psi(\cdot), \tilde{\psi}(\cdot)$ and $M(\cdot)$ denote the functions which were defined in (1), (26) and (22); the sequences $z_{m, n}^{-}$and $z_{m, n}^{+}$represent the poles of $M(\cdot)$ and were defined in (28); the constant $\eta$ is defined by (3).

Definition 2. Define the coefficients $\left\{a_{n}\right\}_{n \geq 0}$ as

$$
a_{n}=-\frac{1}{n !} \prod_{j=0}^{n} \psi(\alpha j), \quad n \geq 0 .
$$

The coefficients $\left\{b_{m, n}\right\}_{m, n \geq 0}$ are defined recursively

$$
\left\{\begin{aligned}
b_{0,0} & =\delta \frac{\Gamma(\eta) \Gamma(-(1-\beta+\gamma) \delta)}{\Gamma(\eta-\hat{\gamma}) \Gamma(-\gamma)} M(1-(1-\beta+\gamma) \delta), \\
b_{m, n} & =-\frac{\psi\left(-\alpha z_{m, n}^{-}\right)}{z_{m, n}^{-}} b_{m, n-1}, \quad m \geq 0, n \geq 1, \\
b_{m, n} & =-\alpha^{\delta(\gamma+\hat{\gamma})} \tilde{\psi}\left(1-\delta-z_{m, n}^{-}\right) \frac{\Gamma\left(z_{m, n}^{-}\right)}{\Gamma\left(z_{m-1, n}^{-}\right)} b_{m-1, n}, \quad m \geq 1, n \geq 0 .
\end{aligned}\right.
$$

Similarly, $\left\{c_{m, n}\right\}_{m, n \geq 0}$ are defined recursively

$$
\left\{\begin{aligned}
c_{0,0} & =\delta \frac{\Gamma(1+\hat{\beta} \delta) \Gamma(1-\beta+\hat{\beta})}{\Gamma(\eta-\hat{\gamma}) \Gamma(\hat{\gamma})} M(\hat{\beta} \delta) \\
c_{m, n} & =-\frac{z_{m, n-1}^{+}}{\psi\left(-\alpha z_{m, n-1}^{+}\right)} c_{m, n-1}, \quad, m \geq 0, n \geq 1, \\
c_{m, n} & =-\frac{\alpha^{-\delta(\gamma+\hat{\gamma})}}{\tilde{\psi}\left(1-\delta-z_{m-1, n}^{+}\right)} \frac{\Gamma\left(z_{m, n}^{+}\right)}{\Gamma\left(z_{m-1, n}^{+}\right)} c_{m-1, n}, \quad m \geq 1, n \geq 0 .
\end{aligned}\right.
$$


Note that if $\beta=1$ we have $\psi(0)=0$, which implies that $a_{n}=0$ for all $n \geq 0$.

Proposition 3. Assume that $\alpha \notin \mathbb{Q}$. For all $m, n \geq 0$ we have

$$
\begin{aligned}
\operatorname{Res}(\mathcal{M}(s): s=-n) & =a_{n}, \quad \text { if } \beta<1 \\
\operatorname{Res}\left(\mathcal{M}(s): s=z_{m, n}^{-}\right) & =b_{m, n}, \\
\operatorname{Res}\left(\mathcal{M}(s): s=z_{m, n}^{+}\right) & =-c_{m, n} .
\end{aligned}
$$

Proof. Let us prove that the residue of $\mathcal{M}(s)$ at $s=z_{m, n}^{-}$is equal to $b_{m, n}$. First, we use Theorem 2 and rearrange the terms in the functional identity (29) to find that

$$
\mathcal{M}(s)=\frac{\delta M(s+1)}{s+(1-\beta+\gamma) \delta} \frac{\Gamma(2-\beta+\gamma+\alpha s) \Gamma(\hat{\beta}+\hat{\gamma}-\alpha s)}{\Gamma(1-\beta+\alpha s) \Gamma(\hat{\beta}-\alpha s)}
$$

The above identity and definition (37) imply that as $s \rightarrow-(1-\beta+\gamma) \delta$

$$
\mathcal{M}(s)=\frac{b_{0,0}}{s+(1-\beta+\gamma) \delta}+O(1)
$$

which means that the residue of $\mathcal{M}(s)$ at $z_{0,0}^{-}=-(1-\beta+\gamma) \delta$ is equal to $b_{0,0}$.

Next, let us prove that the residues satisfy the second recursive identity in (37). We rewrite (29) as

$$
\mathcal{M}(s)=-\frac{\psi(-\alpha s)}{s} \mathcal{M}(s+1)
$$

We know that $\mathcal{M}(s)$ has a simple pole at $s=z_{m, n}^{-}$while $\mathcal{M}(s+1)$ has a simple pole at $z_{m, n}^{-}+1=z_{m-1, n}^{-}$. One can also check that function $\psi(-\alpha s)$ is analytic at $s=z_{m, n}^{-}$for $m \geq 1$. Therefore we have as $s \rightarrow z_{m, n}^{-}$

$$
\begin{aligned}
\mathcal{M}(s) & =\operatorname{Res}\left(\mathcal{M}(s): s=z_{m, n}^{-}\right) \frac{1}{s-z_{m, n}^{-}}+O(1), \\
\mathcal{M}(s+1) & =\operatorname{Res}\left(\mathcal{M}(s): s=z_{m-1, n}^{-}\right) \frac{1}{s-z_{m, n}^{-}}+O(1), \\
-\frac{\psi(-\alpha s)}{s} & =-\frac{\psi\left(-\alpha z_{m, n}^{-}\right)}{z_{m, n}^{-}}+O\left(s-z_{m, n}^{-}\right)
\end{aligned}
$$

which, together with (39) imply that

$$
\operatorname{Res}\left(\mathcal{M}(s): s=z_{m, n}^{-}\right)=-\frac{\psi\left(-\alpha z_{m, n}^{-}\right)}{z_{m, n}^{-}} \times \operatorname{Res}\left(\mathcal{M}(s): s=z_{m-1, n}^{-}\right)
$$

The proof of all remaining cases is very similar and we leave the details to the reader.

Proposition 3 immediately gives us a complete asymptotic expansion of $p(x)$ as $x \rightarrow 0^{+}$and $x \rightarrow+\infty$, presented in the next Theorem. 
Theorem 3. Assume that $\alpha \notin \mathbb{Q}$. Then

$$
\begin{array}{ll}
p(x) \sim \sum_{n \geq 0} a_{n} x^{n}+\sum_{m \geq 0} \sum_{n \geq 0} b_{m, n} x^{(m+1-\beta+\gamma) \delta+n}, & x \rightarrow 0^{+}, \\
p(x) \sim \sum_{m \geq 0} \sum_{n \geq 0} c_{m, n} x^{-(m+\hat{\beta}) \delta-n-1}, & x \rightarrow+\infty .
\end{array}
$$

Proof. The starting point of the proof is the expression of $p(x)$ as the inverse Mellin transform

$$
p(x)=\frac{1}{2 \pi \mathrm{i}} \int_{1+\mathbb{i} \mathbb{R}} \mathcal{M}(s) x^{-s} \mathrm{~d} s, \quad x>0 .
$$

Due to (25), Theorem 2 and Stirling's formula (34) we know that $|\mathcal{M}(x+\mathrm{i} u)|$ decreases exponentially as $u \rightarrow \infty$ (uniformly in $x$ in any finite interval), therefore the integral in the right-hand side of (42) converges absolutely and $p(x)$ is a smooth function for $x>0$. Assume that $c<0$ satisfies $c \neq z_{m, n}^{-}$and $c \neq-n$ for all $m, n$. Shifting the contour of integration $1+\mathrm{i} \mathbb{R} \mapsto c+\mathrm{i} \mathbb{R}$ and taking into account the residues at the poles $s=z_{m, n}^{-}$, we find that

$$
\begin{aligned}
p(x)=\sum \operatorname{Res}\left(\mathcal{M}(s): s=z_{m, n}^{-}\right) \times x^{-z_{m, n}^{-}} & +\sum_{0 \leq n<|c|} \operatorname{Res}(\mathcal{M}(s): s=-n) \times x^{n} \\
& +\frac{1}{2 \pi \mathrm{i}} \int_{c+\mathrm{i} \mathbb{R}} \mathcal{M}(s) x^{-s} \mathrm{~d} s .
\end{aligned}
$$

where the first summation is over all $m \geq 0, n \geq 0$, such that $z_{m, n}^{-}>c$. Next, we perform a change of variables $s=c+\mathrm{i} u$ and obtain the following estimate

$$
\left|\int_{c+i \mathbb{R}} \mathcal{M}(s) x^{-s} \mathrm{~d} s\right|=x^{-c}\left|\int_{\mathbb{R}} \mathcal{M}(s) x^{-\mathrm{i} u} \mathrm{~d} u\right|<x^{-c} \int_{\mathbb{R}}|\mathcal{M}(c+\mathrm{i} u)| \mathrm{d} u=O\left(x^{-c}\right)
$$

which proves (40). The proof of (41) is identical, except that we have to shift the contour of integration in the opposite direction.

It turns out that for almost all parameters $\alpha$ the asymptotic series (40) and (41) converge to $p(x)$ for all $x>0$. In order to state this result, we need to define the following set of real numbers.

Definition 3. Let $\mathcal{L}$ be the set of real irrational numbers $x$, for which there exists a constant $b>1$ such that the inequality

$$
\left|x-\frac{p}{q}\right|<\frac{1}{b^{q}}
$$

is satisfied for infinitely many integers $p$ and $q$.

This set was introduced in [25] in the connection with the distribution of the supremum of a stable process and it was later studied in [23]. It was proved in [23] that $x \in \mathcal{L}$ if and only if

$$
\lim _{q \rightarrow+\infty} \frac{\ln \langle q x\rangle}{q}=0 .
$$


where $\langle x\rangle=\min \{|x-i|: i \in \mathbb{Z}\}$. There also exists a characterization of elements of $\mathcal{L}$ in terms of their continued fraction expansion (see Proposition 1 in [23]). It is known that $\mathcal{L}$ is a proper subset of Liouville numbers, that it is dense in $\mathbb{R}$ and that it has Lebesgue measure zero. The Hausdorff dimension of $\mathcal{L}$ is also zero. This set is closed under addition/multiplication by rational numbers. It is also known that $x \in \mathcal{L}$ if and only if $x^{-1} \in \mathcal{L}$. See [23] for proofs of these results and for some further references.

The following Theorem is our main result in this section.

Theorem 4. Assume that $\alpha \notin \mathcal{L} \cup \mathbb{Q}$. Then for all $x>0$

$$
p(x)= \begin{cases}\sum_{n \geq 0} a_{n} x^{n}+\sum_{m \geq 0} \sum_{n \geq 0} b_{m, n} x^{(m+1-\beta+\gamma) \delta+n}, & \text { if } \gamma+\hat{\gamma}<1, \\ \sum_{m \geq 0} \sum_{n \geq 0} c_{m, n} x^{-(m+\hat{\beta}) \delta-n-1}, & \text { if } \gamma+\hat{\gamma}>1 .\end{cases}
$$

First let us establish the following technical result, which gives us a formula for $M(s)$ similar to the reflection formula for the Gamma function.

Lemma 3. Define

$$
c_{k}=1-(1-\beta+\gamma) \delta-\delta / 2-k
$$

Then for all $u \in \mathbb{C}$

$$
\mathcal{M}\left(c_{k}+\mathrm{i} u\right)=(-1)^{k} \mathcal{M}\left(c_{k}+k+\mathrm{i} u\right) \times \prod_{j=0}^{k-1} \frac{\cos (\pi \alpha(j-\mathrm{i} u+\gamma \delta))}{\cos (\pi \alpha(j-\mathrm{i} u))} \times \frac{F(-\mathrm{i} u)}{F(-\mathrm{i} u+k)}
$$

where we have defined

$$
F(w)=\Gamma((1-\beta+\gamma) \delta+\delta / 2+w) \frac{G\left(\frac{3}{2} \delta+w ; \delta\right)}{G\left(\left(\gamma+\frac{3}{2}\right) \delta+w ; \delta\right)} \times \frac{G\left(\left(\eta-\hat{\gamma}+\frac{1}{2}\right) \delta+w ; \delta\right)}{G\left(\left(\eta+\frac{1}{2}\right) \delta+w ; \delta\right)} .
$$

Proof. Let us set $s=c_{k}+\mathrm{i} u$. Then iterating the functional identity (29) $k$ times we obtain

$$
\mathcal{M}(s)=\mathcal{M}(s+k) \frac{\Gamma(s)}{\Gamma(s+k)} \prod_{j=0}^{k-1} \frac{\Gamma(1-\beta+\gamma+\alpha s+\alpha j)}{\Gamma(1-\beta+\alpha s+\alpha j)} \frac{\Gamma(\hat{\beta}+\hat{\gamma}-\alpha s-\alpha j)}{\Gamma(\hat{\beta}-\alpha s-\alpha j)}
$$

We use identity

$$
\frac{\Gamma(s)}{\Gamma(s+k)}=(-1)^{k} \frac{\Gamma(1-k-s)}{\Gamma(1-s)}
$$

and the reflection formula for the gamma function and rewrite (50) as

$$
\begin{aligned}
\mathcal{M}(s) & =(-1)^{k} \mathcal{M}(s+k) \frac{\Gamma((1-\beta+\gamma) \delta+\delta / 2-\mathrm{i} u)}{\Gamma(k+(1-\beta+\gamma) \delta+\delta / 2-\mathrm{i} u)} \\
& \times \prod_{j=0}^{k-1} \frac{\Gamma\left(-\alpha(k-1-j)-\frac{1}{2}+\mathrm{i} \alpha u\right)}{\Gamma\left(-\alpha(k-1-j)-\gamma-\frac{1}{2}+\mathrm{i} \alpha u\right)} \frac{\Gamma\left(\alpha(k-1-j)+\eta+\frac{1}{2}-\mathrm{i} \alpha u\right)}{\Gamma\left(\alpha(k-1-j)+\eta-\hat{\gamma}+\frac{1}{2}-\mathrm{i} \alpha u\right)} .
\end{aligned}
$$


Next we change the index $j \mapsto k-1-j$ and use reflection formula for the gamma function to obtain

$$
\prod_{j=0}^{k-1} \frac{\Gamma\left(-\alpha(k-1-j)-\frac{1}{2}+\mathrm{i} \alpha u\right)}{\Gamma\left(-\alpha(k-1-j)-\gamma-\frac{1}{2}+\mathrm{i} \alpha u\right)}=\prod_{j=0}^{k-1} \frac{\cos (\pi \alpha(j-\mathrm{i} u+\gamma \delta))}{\cos (\pi \alpha(j-\mathrm{i} u))} \frac{\Gamma\left(\alpha j+\gamma+\frac{3}{2}-\mathrm{i} \alpha u\right)}{\Gamma\left(\alpha j+\frac{3}{2}-\mathrm{i} \alpha u\right)}
$$

The proof of (48) follows from (51), (53) and the following identity

$$
\prod_{j=0}^{k-1} \Gamma(\alpha j+z)=\frac{G(\delta z+k ; \delta)}{G(\delta z ; \delta)}
$$

which is obtained by iterating formula (19) $k$ times.

Proof of Theorem 4: We will use a similar technique as in the proof of Theorem 2 in [23]. Let us define $B=1-\gamma-\hat{\gamma}$ and assume that $B>0$. We start with (43) and set $c=c_{k}$, where $c_{k}$ is defined by (47). Note that $\mathcal{M}(s)$ does not have singularities on the vertical line $c_{k}+\mathrm{i} \mathbb{R}$; if this was not the case then $c_{k}$ would coincide with one of the poles $z_{m, n}^{-}$, which would imply that $\alpha$ is rational.

We define

$$
I_{1}(x, k)=x^{-c_{k}} \operatorname{Re}\left[\int_{0}^{k} \mathcal{M}\left(c_{k}+\mathrm{i} u\right) x^{-\mathrm{i} u} \mathrm{~d} u\right], \quad I_{2}(x, k)=x^{-c_{k}} \operatorname{Re}\left[\int_{k}^{\infty} \mathcal{M}\left(c_{k}+\mathrm{i} u\right) x^{-\mathrm{i} u} \mathrm{~d} u\right] .
$$

It is clear that the integral in the right-hand side of (43) is equal to $2\left(I_{1}(x, k)+I_{2}(x, k)\right)$. Our goal is to prove that $I_{j}(x, k) \rightarrow 0$ as $k \rightarrow+\infty$ for all $x>0$.

First, let us deal with $I_{2}(x, k)$. Using Theorem 2, formula (24) and Stirling's asymptotic formula (34) we find that there exists a constant $C_{1}>0$ such that for all $s$ in the domain

$$
\mathcal{D}=\left\{s \in \mathbb{C}:|s|>2 \text { and } \frac{\pi}{8}<\arg (s)<\frac{7 \pi}{8}\right\}
$$

we have an upper bound

$$
|\mathcal{M}(s)|<|s|^{C_{1}} \exp (\operatorname{Re}[B s \ln (s)+s((1+\ln (\delta))(1-B)+\pi \mathrm{i} \hat{\gamma}-1)]) .
$$

From now on we will denote $u=\operatorname{Im}(s)$. Computing the real part in the above expression we obtain that for all $s \in \mathcal{D}$

$$
|\mathcal{M}(s)|<|s|^{C_{1}} \exp (B \operatorname{Re}(s) \ln |s|-(\pi \hat{\gamma}+B \arg (s)) u+\operatorname{Re}(s)((1+\ln (\delta))(1-B)-1))
$$

Next we check that for all $k$ large enough, conditions $u>k$ and $s=c_{k}+\mathrm{i} u$ imply $s \in \mathcal{D}$, so the integrand in formula (54) defining $I_{2}(x, k)$ can be bounded from above as given in (55). Let us restrict $s$ to the line of integration $L_{2}=\left\{s=c_{k}+\mathrm{i} u: u \geq k\right\}$ and simplify this upper bound. Basically, we want to isolate a term of the form $\exp (-B k \ln (k))$ and show that everything else does not grow faster than an exponential function of $k$. First of all, when $s \in L_{2}$ we have $\operatorname{Re}(s)=c_{k}<1-k$ and $|s|>|\operatorname{Re}(s)|=\left|c_{k}\right|>k-1$, therefore

$$
\exp (B \operatorname{Re}(s) \ln |s|)<\exp (-B(k-1) \ln (k-1))
$$


Next, for $s \in L_{2}$ and $k$ sufficiently large it is true that $-2 k<\operatorname{Re}(s)<1-k$, which implies that there exists a constant $C_{2}>0$ such that

$$
\exp (\operatorname{Re}(s)((1+\ln (\delta))(1-B)-1))<C_{2}^{k}
$$

Finally, for $s \in L_{2}$ we have $\arg (s)>0$, which together with the assumption $B>0$ shows that for all $u>0$

$$
\exp (-(\pi \hat{\gamma}+B \arg (s)) u)<\exp (-\pi \hat{\gamma} u)
$$

Combining the above three estimates with (55) and (54) we see that

$$
\begin{aligned}
\left|I_{2}(x, k)\right| & \leq x^{-c_{k}} \int_{k}^{\infty}\left|\mathcal{M}\left(c_{k}+\mathrm{i} u\right)\right| \mathrm{d} u \\
& <x^{-c_{k}} C_{2}^{k} e^{-B(k-1) \ln (k-1)} \int_{k}^{\infty}\left(c_{k}^{2}+u^{2}\right)^{\frac{C_{1}}{2}} e^{-\pi \hat{\gamma} u} \mathrm{~d} u \\
& <\left|c_{k}\right|^{C_{1}+1} x^{-c_{k}} C_{2}^{k} e^{-B(k-1) \ln (k-1)} .
\end{aligned}
$$

The right-hand side of the above inequality converges to 0 as $k \rightarrow+\infty$, therefore $I_{2}(x, k) \rightarrow 0$ as $k \rightarrow+\infty$.

Now we will deal with $I_{1}(x, k)$. Our first goal is to find an upper bound for the product of trigonometric functions in (48). We will follow the proof of Theorem 2 in [23]: we use the trigonometric identities

$$
\begin{aligned}
\cos (x+\mathrm{i} y) & =\cos (x) \cosh (y)-\mathrm{i} \sin (x) \sinh (y), \\
|\cos (x+\mathrm{i} y)|^{2} & =\cosh (y)^{2}-\sin (x)^{2},
\end{aligned}
$$

which imply that $|\cos (x)| \cosh (y) \leq|\cos (x+\mathrm{i} y)| \leq \cosh (y)$, therefore

$$
\left|\frac{\cos (a+\mathrm{i} y)}{\cos (b+\mathrm{i} y)}\right| \leq \frac{1}{|\cos (b)|} .
$$

Applying the above estimate and Lemma 1 from [23] we conclude that for $\alpha \notin \mathcal{L} \cup \mathbb{Q}$ and for $k$ large enough

$$
\left|\prod_{j=0}^{k-1} \frac{\cos (\pi \alpha(j-\mathrm{i} u+\gamma \delta))}{\cos (\pi \alpha(j-\mathrm{i} u))}\right| \leq \prod_{j=0}^{k-1}|\sec (\pi \alpha j)|=2^{k+o(k)}<3^{k} .
$$

Using (54), (50) and the above inequality we conclude that for all $k$ large enough

$$
\left|I_{1}(x, k)\right|<x^{-c_{k}} 3^{k} \int_{0}^{k}\left|\mathcal{M}\left(c_{k}+k+\mathrm{i} u\right)\right| \times\left|\frac{F(-\mathrm{i} u)}{F(-\mathrm{i} u+k)}\right| \mathrm{d} u .
$$

Now our goal is to prove that $F(\mathrm{i} u) / F(-\mathrm{i} u+k)$ converges to zero faster than any exponential function of $k$ as $k \rightarrow+\infty$. We use (49), Stirling's formula (34) and the asymptotic expansion (23) to conclude that when $w \rightarrow \infty$ in the domain $|\arg (w)|<3 \pi / 4$ we have

$$
\ln (F(w))=B w \ln (w)+O(w) .
$$


This asymptotic result implies that there exists a constant $C_{3}>0$ such that for $k$ large enough and for all $v \in[0,1]$

$$
\begin{aligned}
\left|\frac{F(-\mathrm{i} k v)}{F(-\mathrm{i} k v+k)}\right| & <C_{3}^{k} \exp (B \operatorname{Re}[(-\mathrm{i} k v) \ln (-\mathrm{i} k v)-k(1-\mathrm{i} v) \ln (k(1-\mathrm{i} v))]) \\
& =C_{3}^{k} \exp \left(B\left[-k \ln \left(k \sqrt{1+v^{2}}\right)+k v\left(\arctan (v)-\frac{\pi}{2}\right)\right]\right) \\
& <C_{3}^{k} \exp (-B k \ln (k))
\end{aligned}
$$

where in the last step we have used the fact that $\arctan (v)<\pi / 2$ and $B>0$. Thus we see that for all $k$ large enough we have

$$
\max _{0 \leq u \leq k}\left|\frac{F(-\mathrm{i} u)}{F(-\mathrm{i} u+k)}\right|=\max _{0 \leq v \leq 1}\left|\frac{F(-\mathrm{i} k v)}{F(-\mathrm{i} k v+k)}\right|<C_{3}^{k} \exp (-B k \ln (k))
$$

Combining the above estimate with (57) we obtain that for all $k$ large enough

$$
\left|I_{1}(x, k)\right|<x^{-c_{k}} 3^{k} C_{3}^{k} e^{-B k \ln (k)} \int_{0}^{\infty}|\mathcal{M}(1-(1-\beta+\gamma) \delta-\delta / 2+\mathrm{i} u)| \mathrm{d} u
$$

and we see that $I_{1}(x, k) \rightarrow 0$ as $k \rightarrow+\infty$. Thus when $\gamma+\hat{\gamma}<1$ the first series in (46) converges to $p(x)$ for all $x>0$.

When $\gamma+\hat{\gamma}>1$ the proof is very similar, except that one has to shift the contour of integration in the opposite direction.

\section{Applications}

In this section we will present several applications of the above results on exponential functionals of the hypergeometric Lévy processes. In particular, we will study various functionals related to strictly stable Lévy processes. Our main tool will be the Lamperti transformation, which links a positive self-similar Markov process (pssMp) to an associated Lévy process. By studying this associated Lévy process and its exponential functional we can obtain many interesting results about the original self-similar Markov process.

We assume that the reader is familiar with the Lamperti transformation [30] and Lamperti-stable processes (see $[10,18,13,12])$.

\subsection{Extrema of stable processes}

As our first example, we will present a new proof of some the results on the distribution of extrema of general stable processes, which were obtained in [25] and [23]. Let us define $S_{1}=\sup \left\{Y_{t}: 0 \leq t \leq 1\right\}$, where $Y$ is a stable process with parameters $(\alpha, \rho)$, whose characteristic exponent is given by $(7)$. The infinite series representation for the density of $S_{1}$ (valid for almost all values of $\alpha$ ) was derived recently in [23]. This formula was obtained using an explicit expression for the Mellin transform of $S_{1}$, which was found in [25] via the Wiener-Hopf factorization of stable processes. Our goal in this section is to present a more direct route which leads to the density of $S_{1}$. 
Proposition 4. We have the following identity in distribution

$$
S_{1}^{-\alpha} \stackrel{d}{=} I(\alpha, \xi)
$$

where $\xi$ is a hypergeometric Lévy process with parameters $(\alpha \rho, \alpha \rho, \alpha \rho, \alpha(1-\rho))$.

Proof. Some of the arguments in our proof have been used before in [18], nevertheless, we provide them here for completeness. Let us consider the dual process $\hat{Y}=-Y$. Note that the process $\hat{Y}$ is itself a stable process with parameters $(\alpha, 1-\rho)$. Let $\hat{Z}$ be the process killed at the first exit from the positive half-line

$$
\hat{Z}_{t}=\left\{\begin{array}{cc}
\hat{Y}_{t}, & \text { if } t<\hat{T}, \\
\Delta, & \text { if } t \geq \hat{T},
\end{array}\right.
$$

where $\hat{T}=\inf \left\{t: \hat{Y}_{t} \leq 0\right\}$ and $\Delta$ is the cemetery state. Let $\hat{\xi}$ be the process associated with $\hat{Z}$ by the Lamperti transformation. We use Theorem 1 and find that $\hat{\xi}$ is a hypergeometric process with parameters $(1-\alpha \rho, \alpha(1-\rho), 1-\alpha \rho, \alpha \rho)$.

Next, from the Lamperti transformation we find that $\hat{T} \stackrel{d}{=} I(\alpha,-\hat{\xi})$. This statement is equivalent to $\hat{T} \stackrel{d}{=} I(\alpha, \xi)$, where $\xi=-\hat{\xi}$ is a hypergeometric process with parameters $(\alpha \rho, \alpha \rho, \alpha \rho, \alpha(1-\rho))$ (see Proposition 1(v)). The rest of the proof follows from the chain of identities

$$
\begin{aligned}
\mathbb{P}_{1}(\hat{T}>t) & =\mathbb{P}_{1}\left(\inf _{0 \leq s \leq t} \hat{Y}_{s}>0\right)=\mathbb{P}_{0}\left(\inf _{0 \leq s \leq t} \hat{Y}_{s}>-1\right) \\
& =\mathbb{P}_{0}\left(\sup _{0 \leq s \leq t} Y_{s}<1\right)=\mathbb{P}_{0}\left(t^{1 / \alpha} \sup _{0 \leq s \leq 1} Y_{s}<1\right)=\mathbb{P}_{0}\left(S_{1}^{-\alpha}>t\right) .
\end{aligned}
$$

Proposition 4 implies that the density of $S_{1}$ can be represented in terms of the density of $I(\alpha, \xi)$ (denoted by $p(x)$ ) as follows

$$
\frac{\mathrm{d}}{\mathrm{d} x} \mathbb{P}\left(S_{1} \leq x\right)=\alpha x^{-1-\alpha} p\left(x^{-\alpha}\right), \quad x>0 .
$$

Using this expression and Theorem 3 \{Theorem 4$\}$ we recover the asymptotic expansions that appears in Theorem 9 in [25] \{the convergent series representations given in Theorem 2 in [23]\}.

Remark 2: Note that the convergent and asymptotic series given in Theorems 4 and 3 have identical form. Combining these two results we obtain the following picture: depending on whether $\gamma+\hat{\gamma}<1$ or $\gamma+\hat{\gamma}>1$ one of the series in (46) converges for all $x \in(0, \infty)$, and this convergent series is also asymptotic at one of the boundaries of this interval (at 0 or $+\infty)$. At the same time the second series in (46) provides an asymptotic series at the other boundary. Therefore in what follows we will only present the statements about the convergent series representation, with the understanding that the asymptotic expansions are implicitly imbedded into these results. 


\subsection{Entrance laws}

In this section we will obtain some explicit results on the entrance law of a stable process conditioned to stay positive and the entrance law of the excursion measure of the stable process reflected at its infimum. We will use the following result from [6]: if a non-arithmetic Lévy process $X$ satisfies $\mathbb{E}\left[\left|X_{1}\right|\right]<\infty$ and $\mathbb{E}\left[X_{1}\right]>0$, then as $x \rightarrow 0+$ its corresponding $\operatorname{pssMp}\left(Z, \mathbf{Q}_{x}\right)$ in the Lamperti representation converges weakly (in the sense of finite-dimensional distributions) towards a nondegenerate probability law $\left(Z, \mathbf{Q}_{0}\right)$. Under these conditions, the entrance law under $\mathbf{Q}_{0}$ is described as follows: for every $t>0$ and every measurable function $f: \mathbb{R}_{+} \rightarrow \mathbb{R}_{+}$,

$$
\mathbf{Q}_{0}\left[f\left(Z_{t}\right)\right]=\frac{1}{\alpha \mathbb{E}\left[X_{1}\right]} \mathbb{E}\left[\frac{1}{I} f\left(\frac{t}{I}\right)\right],
$$

where $I=I(\alpha, X)=\int_{0}^{\infty} \exp \left\{-\alpha X_{s}\right\} \mathrm{d} s$. Necessary and sufficient conditions for the weak convergence of $\left(Z, \mathbf{Q}_{x}\right)$ on the Skorokhod space were given in [11].

As before, we consider a stable process $\left(Y, \mathbb{P}_{x}\right)$ and denote by $\left(Y, \mathbb{P}_{x}^{\uparrow}\right)$ the stable process conditioned to stay positive (see $[16,17]$ for a proper definition). It is known that $\left(Y, \mathbb{P}_{x}^{\uparrow}\right)$ is a pssMp with index $\alpha$. According to Corollary 2 in [10] (and Theorem 1 above) its associated Lévy process is given by $\xi^{\uparrow}$, which is a hypergeometric Lévy process with parameters $(1, \alpha \rho, 1, \alpha(1-\rho))$. From Proposition 1 (ii) we see that $\mathbb{E}\left[\xi_{1}^{\uparrow}\right]>0$, and the fact that hypergeometric processes have Lévy measures with exponentially decaying tails implies that $\mathbb{E}\left[\left|\xi_{1}^{\uparrow}\right|\right]<\infty$, thus we have the weak convergence of $\left(Y, \mathbb{P}_{x}^{\uparrow}\right)$ as $x \rightarrow 0^{+}$. We

denote the limiting law by $\mathbb{P}^{\uparrow}$. Note also that in this particular case, the weak convergence of $\left(Y, \mathbb{P}_{x}^{\uparrow}\right)$ has been proved in a direct way in [16].

Everywhere in this section the coefficients $\left\{b_{m, n}\right\}_{m, n \geq 0}$ and $\left\{c_{m, n}\right\}_{m, n \geq 0}$ are defined as in Definition 2 with parameters $\beta=\hat{\beta}=1, \gamma=\alpha \rho$ and $\hat{\gamma}=\alpha(1-\rho)$.

Proposition 5. Let $p_{t}^{\uparrow}$ be the density of the entrance law of $\left(Y, \mathbb{P}^{\uparrow}\right)$. If $\alpha \notin \mathcal{L} \cup \mathbb{Q}$ then for $x>0$ we have

$$
p_{1}^{\uparrow}(x)= \begin{cases}\frac{x^{-1-\rho}}{\alpha \Gamma(\alpha \rho) \Gamma(1+\alpha(1-\rho))} \sum_{m \geq 0} \sum_{n \geq 0} b_{m, n} x^{-m / \alpha-n}, & \text { if } \alpha<1, \\ \frac{x^{1 / \alpha}}{\alpha \Gamma(\alpha \rho) \Gamma(1+\alpha(1-\rho))} \sum_{m \geq 0} \sum_{n \geq 0} c_{m, n} x^{m / \alpha+n}, & \text { if } \alpha>1 .\end{cases}
$$

Proof. Let $p(x)$ be the density of $I\left(\alpha, \xi^{\uparrow}\right)$. From (59) we find that the density of the entrance law of $\left(Y, \mathbb{P}^{\uparrow}\right)$ is given by

$$
p_{1}^{\uparrow}(x)=\frac{1}{\alpha \mathbb{E}\left[\xi_{1}^{\uparrow}\right]} x^{-1} p\left(x^{-1}\right) .
$$

Using Proposition 1 (ii) we obtain $\mathbb{E}\left[\xi_{1}^{\uparrow}\right]=\Gamma(\alpha \rho) \Gamma(1+\alpha(1-\rho))$. In order to finish the proof we only need to apply results of Theorem 46.

Proposition 6. Let $q_{t}$ be the density of the entrance law of the excursion measure of the reflected process $(Y-\underline{Y}, \mathbb{P})$, where $\underline{Y}_{t}=\inf _{0 \leq s \leq t} Y_{s}$. If $\alpha \notin \mathcal{L} \cup \mathbb{Q}$ then for $x>0$ we have

$$
q_{1}(x)= \begin{cases}\frac{x^{-1-\rho-\alpha(1-\rho)}}{\alpha \Gamma(\alpha \rho) \Gamma(1+\alpha(1-\rho))} \sum_{m \geq 0} \sum_{n \geq 0} b_{m, n} x^{-m / \alpha-n}, & \text { if } \alpha<1, \\ \frac{x^{1 / \alpha-\alpha(1-\rho)}}{\alpha \Gamma(\alpha \rho) \Gamma(1+\alpha(1-\rho))} \sum_{m \geq 0} \sum_{n \geq 0} c_{m, n} x^{m / \alpha+n}, & \text { if } \alpha>1 .\end{cases}
$$


Proof. The proof follows from Proposition 5 and Theorem 3 in [16], which gives us the identity $q_{1}=$ $x^{-\alpha(1-\rho)} p_{1}^{\uparrow}(x)$.

\subsection{The distribution of the lifetime of a stable process conditioned to hit zero continuously}

Our third application deals with stable processes conditioned to hit zero continuously. Processes in this class are defined as Doob's $h$-transform with respect to the function $h(x)=x^{\alpha(1-\rho)-1}$, which is excessive for the killed process $\left(Y_{t} \mathbf{1}_{\{t<T\}}, \mathbb{P}_{x}\right)$. Its law $\mathbb{P}_{x}^{\downarrow}$, which is defined on each $\sigma$-field $\mathcal{F}_{t}$ by

$$
\left.\frac{\mathrm{d} \mathbb{P}_{x}^{\downarrow}}{\mathrm{d} \mathbb{P}_{x}}\right|_{\mathcal{F}_{t}}=\frac{Y_{t}^{\alpha(1-\rho)-1}}{x^{\alpha(1-\rho)-1}} \mathbf{1}_{\{t<T\}}
$$

is that of a pssMp that hits zero in a continuous way. According to Corollary 3 in [10] (and Theorem 1 above), its associated Lévy process in the Lamperti representation is the hypergeometric Lévy process $\xi^{\downarrow}$ with parameters $(0, \alpha \rho, 0, \alpha(1-\rho))$. In particular from Proposition 1(ii) we find that the process $\xi^{\downarrow}$ drifts to $-\infty$. Let $T^{\downarrow}$ be the life-time of the stable process conditioned to hit zero continuously. From the Lamperti transformation it follows that $T^{\downarrow} \stackrel{d}{=} I\left(\alpha,-\xi^{\downarrow}\right)$, therefore $T^{\downarrow} \stackrel{d}{=} I(\alpha, \xi)$, where $\xi$ is a hypergeometric process with parameters $(1, \alpha(1-\rho), 1, \alpha \rho)$. Let us define the coefficients $\left\{b_{m, n}\right\}_{m, n \geq 0}$ and $\left\{c_{m, n}\right\}_{m, n \geq 0}$ as in Definition 2 with $\beta=\hat{\beta}=1, \gamma=\alpha(1-\rho)$ and $\hat{\gamma}=\alpha \rho$. Then according to Theorem 46, if $\alpha \notin \mathcal{L} \cup \mathbb{Q}$ and $x>0$ we have

$$
\frac{\mathrm{d}}{\mathrm{d} t} \mathbb{P}_{1}^{\downarrow}\left(T^{\downarrow} \leq t\right)= \begin{cases}t^{1-\rho} \sum_{m \geq 0} \sum_{n \geq 0} b_{m, n} t^{m / \alpha+n}, & \text { if } \alpha<1, \\ t^{-1-1 / \alpha} \sum_{m \geq 0} \sum_{n \geq 0} c_{m, n} t^{-m / \alpha-n}, & \text { if } \alpha>1 .\end{cases}
$$

\subsection{Distribution of some functionals related to the radial part of a sym- metric stable process}

Our last application deals with the radial part of symmetric stable processes in $\mathbb{R}^{d}$. Let $\mathbf{Y}=\left(\mathbf{Y}_{t}, t \geq 0\right)$ be a symmetric stable Lévy process of index $\alpha \in(0,2)$ in $\mathbb{R}^{d}(d \geq 1)$, defined by

$$
\mathbb{E}_{0}\left[e^{i\left\langle\lambda, \mathbf{Y}_{t}\right\rangle}\right]=e^{-t\|\lambda\|^{\alpha}}
$$

for all $t \geq 0$ and $\lambda \in \mathbb{R}^{d}$. Here $\mathbb{P}_{y}$ denotes the law of the process $\mathbf{Y}$ started from $y \in \mathbb{R}^{d},\|\cdot\|$ is the norm in $\mathbb{R}^{d}$ and $\langle\cdot, \cdot\rangle$ is the Euclidean inner product.

According to Caballero et al. [12], when $\alpha<d$ the radial process $R_{t}=\frac{1}{2}\left\|\mathbf{Y}_{t}\right\|$ is a transient positive self-similar Markov process with index $\alpha$ and infinite lifetime. From Theorem 7 in [12] we find that the Laplace exponent of its associated Lévy process $\xi$ is given by

$$
\psi_{\xi}(z)=-\frac{\Gamma((\alpha-z) / 2)}{\Gamma(-z / 2)} \frac{\Gamma((z+d) / 2)}{\Gamma((z+d-\alpha) / 2)} .
$$

This shows that the process $X:=2 \xi$ is a hypergeometric Lévy process with parameters $(1, \alpha / 2,(d-$ $\alpha) / 2, \alpha / 2)$. From Proposition 1(ii) we see that $\xi$ drifts to $+\infty$ and that

$$
\mathbb{E}\left[\xi_{1}\right]=\frac{1}{2} \frac{\Gamma\left(\frac{\alpha}{2}\right) \Gamma\left(\frac{d}{2}\right)}{\Gamma\left(\frac{d-\alpha}{2}\right)} .
$$


Let $\mathbf{P}_{x}$ denotes the law of the process $R$ starting from $x>0$. The process $\xi$ satisfies $\mathbb{E}\left[\xi_{1}\right]>0$ and $\mathbb{E}\left[\left|\xi_{1}\right|<\infty\right.$, and according to $[6,11]$ we have the weak convergence of $\left(R, \mathbf{P}_{x}\right)$ towards $\left(R, \mathbf{P}_{0}\right)$ as $x \rightarrow 0^{+}$.

Proposition 7. Let $\tilde{p}_{t}$ be the density of the entrance law of $\left(R, \mathbf{P}_{0}\right)$. If $\alpha<d$ then for $x>0$ we have

$$
\tilde{p}_{1}(x)= \begin{cases}\frac{2 x^{-1}}{\pi \alpha \Gamma\left(\frac{d}{2}\right)} \sum_{n \geq 1} \sin \left(\frac{\pi \alpha n}{2}\right) \Gamma\left(1+\frac{\alpha n}{2}\right) \Gamma\left(\frac{d+\alpha n}{2}\right) \frac{(-1)^{n+1}}{n !} x^{-n}, & \text { if } \alpha<1, \\ \frac{4 x^{-1+d / \alpha}}{\alpha^{2} \Gamma\left(\frac{d}{2}\right)} \sum_{m \geq 0} \frac{\Gamma\left(\frac{d+2 m}{\alpha}\right)}{\Gamma\left(\frac{d+2 m}{2}\right)} \frac{(-1)^{m}}{m !} x^{2 m / \alpha} & \text { if } \alpha>1 .\end{cases}
$$

Proof. We follow the same approach as in the proof of Proposition 5. Using (59) we conclude that $\tilde{p}_{1}(x)=\left(2 / \alpha \mathbb{E}\left[\xi_{1}\right]\right) x^{-1} p\left(x^{-1}\right)$, where $p(x)$ is the density of $I(\alpha, \xi)=I(\alpha / 2, X)$. In the case $\alpha \notin \mathcal{L} \cup \mathbb{Q}$ the infinite series representation for $p(x)$ and the expression for the coefficients follow from Theorem 46 and Definition 2 with $\beta=1, \gamma=\alpha / 2, \hat{\beta}=(d-\alpha) / 2$ and $\hat{\gamma}=\alpha / 2$. Note that both infinite series in (65) converge for all $\alpha$, thus we can remove the assumption $\alpha \notin \mathcal{L} \cup \mathbb{Q}$.

There is also a simpler way to derive an explicit series representation for $p(x)$. Theorem 2 and Definition 1 tell us that the Mellin transform of $I(\alpha / 2, X)$ is given by

$$
\mathbb{E}\left[I(\alpha / 2, X)^{s-1}\right]=\Gamma(s) M\left(s ; \frac{\alpha}{2}, 1, \frac{\alpha}{2}, \frac{d-\alpha}{2}, \frac{\alpha}{2}\right)=C \Gamma(s) \frac{G\left(s ; \frac{2}{\alpha}\right)}{G\left(1+s ; \frac{2}{\alpha}\right)} \frac{G\left(\frac{d}{\alpha}+1-s ; \frac{2}{\alpha}\right)}{G\left(\frac{d}{\alpha}-s ; \frac{2}{\alpha}\right)},
$$

where $C$ is a constant such that the right-hand side of the above identity is equal to one for $s=1$. Using the functional identity (19) for the double gamma function we simplify the right-hand side in (66) and obtain

$$
\mathbb{E}\left[I(\alpha / 2, X)^{s-1}\right]=\frac{\Gamma\left(\frac{\alpha}{2}\right)}{\Gamma\left(\frac{d-\alpha}{2}\right)} \Gamma(s) \frac{\Gamma\left(\frac{d-\alpha s}{2}\right)}{\Gamma\left(\frac{\alpha s}{2}\right)} .
$$

We see that $\mathbb{E}\left[I(\alpha / 2, X)^{s-1}\right]$ has simple poles at points $\{-n\}_{n \geq 1}$ and $\{(d+2 m) / \alpha\}_{m \geq 0}$. The residues at these points (which give us the coefficients in (65)) can be easily found using the fact that the residue of $\Gamma(s)$ at $s=-n$ is equal to $(-1)^{n} / n$ !.

Next, we will study the last passage time of $\left(\mathbf{Y}, \mathbb{P}_{0}\right)$ from the sphere in $\mathbb{R}^{d}$ of radius $r$, i.e.

$$
U_{r}=\sup \left\{s \geq 0:\left\|\mathbf{Y}_{s}\right\|<r\right\} \text {. }
$$

Due to the self-similarity property of $Y$ we find that random variables $U_{r}$ satisfy the scaling property $b^{\alpha} U_{r} \stackrel{d}{=} U_{b r}$ (valid for any $b, r>0$ ), thus it is sufficient to consider the case of of $U_{2}$.

Proposition 8. If $\alpha<d$ then

$$
\frac{\mathrm{d}}{\mathrm{d} t} \mathbb{P}\left(U_{2} \leq t\right)= \begin{cases}\frac{1}{\pi \Gamma\left(\frac{d-\alpha}{2}\right)} \sum_{n \geq 0} \sin \left(\frac{\pi \alpha(1+n)}{2}\right) \Gamma\left(\frac{\alpha(1+n)}{2}\right) \Gamma\left(\frac{d+\alpha n}{2}\right) \frac{(-1)^{n}}{n !} t^{n}, & \text { if } \alpha<1, \\ \frac{2 t^{-d / \alpha}}{\alpha \Gamma\left(\frac{d-\alpha}{2}\right)} \sum_{m \geq 0} \frac{\Gamma\left(\frac{d+2 m}{\alpha}\right)}{\Gamma\left(\frac{d-\alpha}{2}+m+1\right)} \frac{(-1)^{m}}{m !} t^{-2 m / \alpha}, & \text { if } \alpha>1 .\end{cases}
$$

Proof. Let us define the last passage time of $R$ as $L_{r}=\sup \left\{s \geq 0:\left\|R_{s}\right\|<r\right\}$. It is clear that $U_{2} \stackrel{d}{=} L_{1}$. According to Proposition 1 in [19], the random variable $L_{1}$ has the same law as $\mathcal{G}^{\alpha} I(\alpha, \xi)$, where $\xi$ is the 
Lévy process associated to $R$ by the Lamperti transformation and $\mathcal{G}$ is independent of $\xi$. Lemma 1 in [19] tells us that $\mathcal{G} \stackrel{d}{=} e^{-\mathcal{U Z}}$, where $\mathcal{U}$ and $\mathcal{Z}$ are independent random variables, such that $\mathcal{U}$ is uniformly distributed over $[0,1]$ and the law of $\mathcal{Z}$ is given by

$$
\mathbb{P}(\mathcal{Z}>u)=\frac{1}{\mathbb{E}\left[H_{1}\right]} \int_{u}^{\infty} x \nu(\mathrm{d} x), \quad u \geq 0
$$

where $H=\left(H_{t}, t \geq 0\right)$ denotes the ascending ladder height of $\xi$ and $\nu$ its Lévy measure. From the proof of Lemma 3 in [12] we know that $H$ has no linear drift and that its Lévy measure is given by (up to a multiplicative constant)

$$
\nu(\mathrm{d} x)=\frac{e^{2 x}}{\left(e^{2 x}-1\right)^{1+\alpha / 2}} \mathrm{~d} x
$$

Using the above expression, integration by parts, the integral identity (14) and reflection formula for the gamma function we obtain

$$
\mathbb{E}\left[H_{1}\right]=\int_{0}^{\infty} x \nu(\mathrm{d} x)=\frac{\pi}{\alpha \sin \left(\frac{\pi \alpha}{2}\right)} .
$$

Let us find the Mellin transform of $L_{1}$. We use the independence of $U$ and $\mathcal{Z}$ and obtain

$$
\begin{aligned}
\mathbb{E}\left[\mathcal{G}^{s}\right] & =\mathbb{E}\left[e^{-s \mathcal{U} \mathcal{Z}}\right]=\frac{1}{s} \mathbb{E}\left[\frac{1-e^{-s \mathcal{Z}}}{\mathcal{Z}}\right]=\frac{1}{s \mathbb{E}\left[H_{1}\right]} \int_{0}^{\infty} \frac{\left(1-e^{-s z}\right) e^{2 z}}{\left(e^{2 z}-1\right)^{1+\alpha / 2}} \mathrm{~d} z \\
& =-\frac{1}{\alpha s \mathbb{E}\left[H_{1}\right]} \int_{0}^{\infty}\left(1-e^{-s z}\right) \mathrm{d}\left[\left(e^{2 z}-1\right)^{-\alpha / 2}\right] \\
& =\frac{1}{\alpha \mathbb{E}\left[H_{1}\right]} \int_{0}^{\infty} \frac{e^{-s z}}{\left(e^{2 z}-1\right)^{\alpha / 2}} \mathrm{~d} z=\frac{1}{\alpha \mathbb{E}\left[H_{1}\right]} \frac{\Gamma\left(\frac{\alpha}{2}(1+s)\right) \Gamma\left(1-\frac{\alpha}{2}\right)}{\Gamma\left(1+\frac{\alpha s}{2}\right)},
\end{aligned}
$$

where we have again applied integration by parts and integral identity (14). Combining the above two expressions with (67) we have

$$
\mathbb{E}\left[L_{1}^{s-1}\right]=\mathbb{E}\left[\mathcal{G}^{s-1}\right] \times \mathbb{E}\left[I(\alpha, \xi)^{s-1}\right]=\frac{\Gamma(s)}{\Gamma\left(\frac{d-\alpha}{2}\right)} \frac{\Gamma\left(\frac{d-\alpha s}{2}\right)}{\Gamma\left(1-\frac{\alpha(1-s)}{2}\right)} .
$$

The function in the right-hand side of the above equation has simple poles at points $\{-n\}_{n \geq 0}$ and $\{(d+2 m) / \alpha\}_{m \geq 0}$. We express the density of $L_{1}$ as the inverse Mellin transform, compute the residues and use similar technique as in the proof of Theorem 46 to obtain the series representation (68). 


\section{References}

[1] M. Abramowitz And I. A. Stegun, eds., Handbook of mathematical functions: with formulas, graphs, and mathematical tables, Dover Publications, 1970.

[2] E.W. BARnes, The genesis of the double gamma function, Proc. London Math. Soc., 31 (1899), pp. 358-381.

[3] — The theory of the double gamma function, Phil. Trans. Royal Soc. London (A), 196 (1901), pp. 265-387.

[4] V. Bernyk, R.C. Dalang, And G. Peskir, The law of the supremum of a stable Lévy process with no negative jumps, Ann. Probab., 36 (2008), pp. 1777-1789.

[5] J. Bertoin, Lévy processes, vol. 121 of Cambridge Tracts in Mathematics, Cambridge University Press, Cambridge, 1996.

[6] J. Bertoin AND M. Yor, The entrance laws of self-similar Markov processes and exponential functionals of Lévy processes, Potential Anal., 17 (2002), pp. 389-400.

[7] — Exponential functionals of Lévy processes, Probab. Surv., 2 (2005), pp. 191-212 (electronic).

[8] J. Billingham And A.C. KIng, Uniform asymptotic expansions for the Barnes double gamma function, Proc. R. Soc. Lond. A, 453 (1997), pp. 1817-1829.

[9] M. Caballero and V. Rivero, On the asymptotic behaviour of increasing self-similar Markov processes, Electron. J. Probab., 14 (2009), pp. 865-894 (electronic).

[10] M. E. Caballero And L. Chaumont, Conditioned stable Lévy processes and the Lamperti representation, J. Appl. Probab., 43 (2006), pp. 967-983.

[11] — Weak convergence of positive self-similar Markov processes and overshoots of Lévy processes, Ann. Probab., 34 (2006), pp. 1012-1034.

[12] M.E. Caballero, J.C. Pardo, And J.L. Perez, Explicit identities for Lévy processes associated to symmetric stable processes, preprint, (2010).

[13] —, On Lamperti stable processes, Probab. Math. Statist., 30 (2010), pp. 1-28.

[14] N. Cai And S.G. Kou, Prising Asian options under a general jump diffusion model, to appear in Operations Research, (2010).

[15] P. Carmona, F. Petit, And M. Yor, On the distribution and asymptotic results for exponential functionals of Lvy processes, In: M. Yor, Editor, Exponential Functionals and Principal Values Related to Brownian Motion, Bibl. Rev. Mat. Iberoamericana, (1997), pp. 73-121.

[16] L. Chaumont, Conditionings and path decompositions for Lévy processes, Stochastic Process. Appl., 64 (1996), pp. 39-54.

[17] L. Chaumont and R. A. Doney, On Lévy processes conditioned to stay positive, Electron. J. Probab., 10 (2005), pp. no. 28, 948-961 (electronic). 
[18] L. Chaumont, A. E. Kyprianou, And J. C. Pardo, Some explicit identities associated with positive self-similar Markov processes, Stochastic Process. Appl., 119 (2009), pp. 980-1000.

[19] L. Chaumont And J. C. Pardo, The lower envelope of positive self-similar Markov processes, Electron. J. Probab., 11 (2006), pp. no. 49, 1321-1341 (electronic).

[20] J.B. Conway, Functions of one complex variable, Springer-Verlag, 2 ed., 1978.

[21] R.A. Doney, On Wiener-Hopf factorisation and the distribution of extrema for certain stable processes, Ann. Prob., 15 (1987), pp. 1352-1362.

[22] B. HAAs, Loss of mass in deterministic and random fragmentations, Stochastic Process. Appl., 106 (2003), pp. 245-277.

[23] F. Hubalek and A. Kuznetsov, A convergent series representation for the density of the supremum of a stable process, preprint, (2010).

[24] A. Jeffrey, ed., Table of integrals, series and products, Academic Press, 7 ed., 2007.

[25] A. Kuznetsov, On extrema of stable processes, to appear in Ann. Probab., (2010).

[26] A. Kuznetsov, A.E. Kyprianou, J.C. Pardo, and K. Van Schaik, A Wiener-Hopf MonteCarlo simulation technique for Lévy processes, to appear in Ann. Appl. Probab., (2010).

[27] A. Kyprianou, Introductory Lectures on Fluctuations of Lévy Processes with Applications, Springer, 2006.

[28] A. E. Kyprianou And J. C. PARdo, Continuous-state branching processes and self-similarity, J. Appl. Probab., 45 (2008), pp. 1140-1160.

[29] A.E. Kyprianou, J.C. Pardo, And V. M. Rivero, Exact and asymptotic n-tuple laws at first and last passage, Ann. Appl. Probab., 20 (2010), pp. 522-564.

[30] J. LAmperti, Semi-stable Markov processes. I, Z. Wahrscheinlichkeitstheorie und Verw. Gebiete, 22 (1972), pp. 205-225.

[31] J.B. LAWRIE AND A.C. KING, Exact solutions to a class of functional difference equations with application to a moving contact line flow, Euro. J. Appl. Math., 5 (1994), pp. 141-147.

[32] K. Maulik AND B. ZWART, Tail asymptotics for exponential functionals of Lévy processes, Stoch. Proc. Appl., 116 (2006), pp. 156-177.

[33] J. C. PARdo, On the future infimum of positive self-similar Markov processes, Stochastics, 78 (2006), pp. 123-155.

[34] P. PATiE, Exponential functionals of a new family of Lévy processes and self-similar continuous state branching processes with immigration, Bull. Sci. Math., 133 (2009), pp. 355-382.

[35] P. Patie, A few remarks on the supremum of stable processes, Statist. Probab. Lett., 79 (2009), pp. 1125-1128.

[36] P. PATIE, Law of the absorption time of positive self-similar Markov processes, preprint, (2009). 
[37] V. Rivero, A law of iterated logarithm for increasing self-similar Markov processes, Stoch. Stoch. Rep., 75 (2003), pp. 443-472.

[38] _ Recurrent extensions of self-similar Markov processes and Cramér's condition, Bernoulli, 11 (2005), pp. 471-509.

[39] — Recurrent extensions of self-similar Markov processes and Cramér's condition. II, Bernoulli, 13 (2007), pp. 1053-1070.

[40] V. Vigon, Simplifiez vos Lévy en titillant la factorisation de Wiener-Hopf., Thèse de doctorat de l'INSA de Rouen, 2002. 